\title{
Battue ou chasse à courre, en Somalie ou en Asie
}

La traque aux pirates est lancée!

\section{Eric Frécon}

\section{(2) OpenEdition}

Journals

Édition électronique

URL : http://journals.openedition.org/echogeo/10403

DOI : $10.4000 /$ echogeo.10403

ISSN : 1963-1197

\section{Éditeur}

Pôle de recherche pour l'organisation et la diffusion de l'information géographique (CNRS UMR 8586)

\section{Référence électronique}

Eric Frécon, « Battue ou chasse à courre, en Somalie ou en Asie», EchoGéo [En ligne], Sur le Vif, mis en ligne le 09 janvier 2009, consulté le 30 avril 2019. URL : http://journals.openedition.org/ echogeo/10403; DOI : 10.4000/echogeo.10403

Ce document a été généré automatiquement le 30 avril 2019.

\section{(c) (i) () $९$}

EchoGéo est mis à disposition selon les termes de la licence Creative Commons Attribution - Pas d'Utilisation Commerciale - Pas de Modification 4.0 International 


\title{
Battue ou chasse à courre, en Somalie ou en Asie
}

La traque aux pirates est lancée!

\author{
Eric Frécon
}

1 Quatre attaques dans les détroits de Malacca et Singapour lors des trois premiers trimestres 2008, contre 30 en $2003 ; 63$ abordages entre janvier et septembre 2008 au large de la Somalie et dans le Golfe d'Aden contre seulement 31 cinq ans plus tôt $t^{1}:$ l'œil du cyclone pirate a quitté cette année les côtes malaises pour frapper celles de la Corne de l'Afrique. Diplomates et armateurs s'interrogent aujourd'hui sur les mesures à adopter pour contrer ce fléau.

\section{Battue désordonnée et inefficace au large de la Somalie}

2 Après avoir longtemps mésestimé cette menace asymétrique, la communauté internationale semble démunie et attaque en désordre. Jugée obsolète en 2007, une loi datant du XIX siècle et traitant de la piraterie a par exemple été effacée du dispositif pénal français ; six mois plus tard, la Marine nationale devait pourtant réagir avec force contre les pirates, en usant des largesses du droit international maritime, pour récupérer les otages du Ponant. Sur cet élan, l'Union européenne (UE) a emboîté le pas en décembre 2008 avec trois frégates et deux patrouilleurs aériens dédiés à l'escorte de navires vulnérables mais largement insuffisants pour couvrir une surface quatre fois supérieure à celle de la France. La Task Force 150 des alliés n'est pas en reste mais son mandat initial demeure limité à la lutte anti-terroriste et non anti-pirate ; de plus, son idée de corridor soi-disant sécurisé permettait de grouper toutes les proies potentielles, facilitant ainsi la tâche des pirates! Bien qu'arrivée en renfort, l'OTAN (Organisation du traité de l'Atlantique-Nord) se limite à la surveillance des bateaux du Programme alimentaire mondial. Quant à l'ONU (Organisation des Nations unies), elle alimente le débat au fil des délibérations du Conseil de sécurité. Sous cet amas de résolutions, lois, décrets et 
décisions, on en viendrait à oublier les conventions de Montego Bay (1982) et de Rome (1988) censées régir le droit et la sûreté maritimes. En sus, d'autres acteurs interviennent unilatéralement comme le Danemark qui a dû relâcher des pirates faute d'un arsenal juridique adéquat pour lancer une procédure pénale. L'Inde aussi veille sur son océan mais son excès de zèle lui a valu de tirer sur un bateau de pêche thaïlandais qu'elle croyait être un bateau-mère pirate. Coréens, Malaisiens et Russes sont aussi de la partie. Enfin, le 18 décembre 2008, la Chine annonçait sa prochaine implication pour participer à l'effort de lutte.

3 Toute la planète se retrouve au chevet de la Somalie jusqu'ici complètement oubliée. Mais l'accumulation de ces soudaines bonnes volontés conduit à un enchevêtrement juridique dans lequel s'emmêlent toutes les marines sur zone ; pirates pour les uns, terroristes en puissance pour les autres : le régime juridique appliqué aux forbans interpelés, arrêtés ou appréhendés ne sera pas toujours le même. Pour éviter de possibles dérives, la France réfléchit à un projet de loi pour encadrer ses opérations de police maritime, typiques de l'action de l'Etat en mer à laquelle est rompue la Marine nationale.

\section{Embouteillage naval et d'intérêts concurrents dans la Corne de l'Afrique}

4 Ces débauches d'efforts cachent aussi d'autres intérêts plus ou moins avoués. Car au premier abord, ces déploiements, avec la logistique qu'ils nécessitent, coûteront très cher, bien plus que le montant des rançons payées et estimées aujourd'hui à 120 millions de dollars pour les onze premiers mois de $2008^{2}$. Par ailleurs, toute l'économie mondiale ne dépend pas du Golfe d'Aden : y transitaient par jour, en 2006, 3,3 millions de barils de pétrole par jour contre 15 millions dans le détroit de Malacca $^{3}$. De plus, le nombre d'attaques probable pour 2008 (environ 150) rapporté au nombre de navires empruntant cette voie maritime (au minimum 16000 par an) invite à la nuance : moins de $1 \%$ de bateaux sont victimes d'incidents liés à la piraterie, en incluant dans ces chiffres les simples tentatives. Les flux maritimes ne risquent donc pas encore d'être subitement stoppés.

5 Il faut alors chercher ailleurs d'autres explications pour justifier ces efforts. A travers cette première opération navale européenne, Nicolas Sarkozy tenait sans doute à fournir une illustration éclatante du potentiel de l'UE avant de prochaines négociations avec l'OTAN. Cette dernière a également saisie cette occasion pour mettre en place la première opération navale de son histoire. Quant à l'Inde et la Chine, elles se disputent la suprématie régionale. Pour la première fois depuis sa création en 1949, la marine de la Chine populaire quitte sa posture uniquement côtière pour tester ses bâtiments et faire flotter son pavillon plus au large. La démarche n'a rien d'anodin quand on connait ses programmes d'armements pour, à terme, disposer de trois flottes océaniques ${ }^{4}$.

Mais pendant que les diplomates verbiagent, que les stratèges pérorent et que les juristes discourent, les pirates s'activent, de plus en plus loin de leurs côtes et contre des navires de plus en plus gros. Et pendant que les pirates s'activent en Somalie, leurs collègues ou frères des côtes de Sumatra se terrent, eux, dans leurs repaires. Autrefois qualifié de « zone de risque de guerre » par la compagnie Lloyd's jusqu'en 2006, le détroit de Malacca à présent sécurisé fait figure d'exemple. Experts et conseillers y cherchent quelques 
raisons d'espérer et d'éventuelles mesures à imiter - pirater ?! - en Afrique orientale. Une telle démarche se justifie-t-elle ? Pas sûr.

\section{Chasse à courre indonésienne jusque dans les mangroves du détroit de Malacca}

$7 \quad$ En premier lieu, les Asiatiques évoquent volontiers les patrouilles créées pour supprimer la menace pirate de leurs côtes. En 2004, pour se prémunir d'éventuelles manœuvres de surveillance américaines, Indonésiens, Malaisiens et Singapouriens ont mis en place des patrouilles navales suivies, un an plus tard, d'une veille aérienne baptisée Eyes in the Sky. Lancées à grand renfort de campagnes de presse pour rassurer la communauté internationale et maritime, il n'a par la suite jamais été possible de connaitre avec précision les forces impliquées dans cette surveillance côtière: un diplomate a par exemple parlé de seulement trois patrouilles aériennes de trois heures en l'espace de six mois en 2006. Aujourd'hui, on évoque huit rotations par semaine, ce qui est largement insuffisant pour couvrir la zone. Surtout, il paraît compliqué de distinguer, de nuit, à plusieurs milliers de pieds d'altitude, une embarcation de pirates de celle de pêcheurs. De même, un officier de la marine indonésienne avouait qu'envoyer des navires de gros tonnages pour traquer les pirates cachés au plus profond des mangroves, parmi les quelque 17000 les îles et îlots de l'archipel indonésien, se révélait guère judicieux.

Pêcheurs et bateau-taxi à Belakang-Padang, l'un des principaux repaires pirates du détroit de Malacca

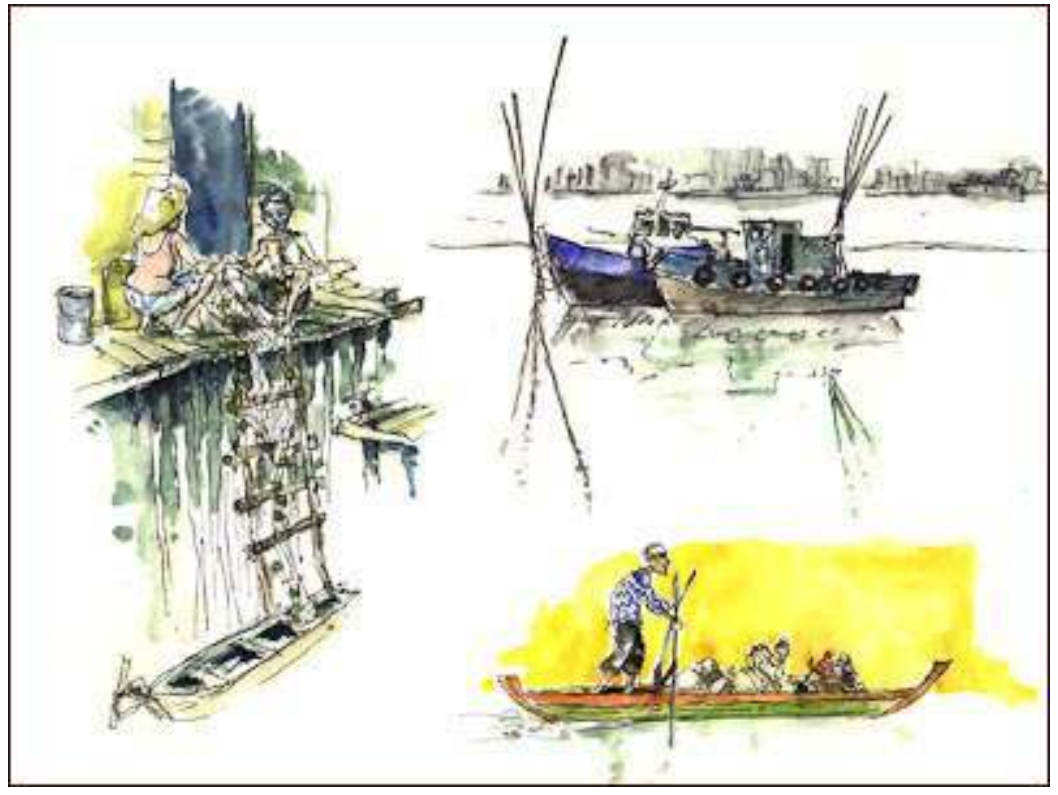

Aquarelle de Sébatien Brunel, 2002-2004 : http://seb.brunel.free.fr

8 En réalité, c'est à l'Indonésie et aux mesures prises à terre que l'on doit en grande partie le récent nettoyage du détroit. Longtemps montré du doigt à juste titre pour avoir hébergé nombre de gangs locaux ou régionaux, l'Etat a réagi en reprenant pied dans ces anciennes zones de non-droit. A force d'infrastructures - comme cette route goudronnée, ces égouts et ce poste de douane sur l'île de Karimun - le gouvernement a réinvesti les ghettos sur pilotis où naissaient les vocations de pirates amers à l'ombre des buildings 
rutilants de l'arrogante Singapour. De même, la nouvelle organisation administrative indonésienne marquée par la création de provinces - comme celle de l'archipel des Riau à l'extrémité sud du détroit de Malacca - a donné davantage de moyens aux forces postées aux confins de l'archipel. Etats-Unis, Japon et Pays-Bas ont complété les efforts en offrant du matériel à une marine indonésienne très pauvre au lendemain de la crise de 1997. Par ailleurs, la stabilisation de la scène politique nationale, après le choc de 1998 et le départ de président Suharto, a également contribué à la pacification des esprits. Enfin, dernière cause du déclin de la piraterie dans la zone : le départ à la retraite des pirates conjugué au développement économique de Batam; les vétérans de Belakang Padang, un ancien hub pirate réputé, expliquent volontiers : « nous avons une famille et ne voulons plus prendre de risques pour partir à l'abordage des navires ; quant à nos enfants, ils ont eu la chance, eux, d'avoir une scolarité. A présent, ils aspirent à un emploi de bureau dans une des nombreuses sociétés occidentales installées dans la zone franche des Riau».

Scènes de la vie quotidienne à Belakang Padang, sous l'œil énigmatique d'un orang Buton

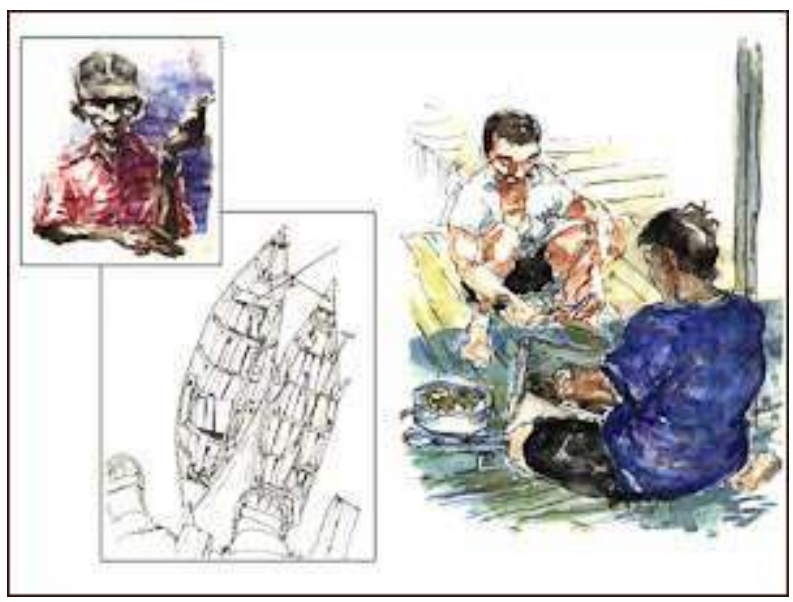

C'est parmi cette ethnie que l'on trouve les pirates les plus craints du repaire. Aquarelle de Sébatien Brunel, 2002-2004 : http://seb.brunel.free.fr

Education, transport, électricité, sécurité et autres fonctions régaliennes: l'Etat est progressivement de retour sur les côtes indonésiennes du détroit de Malacca. Lui plus que les seules patrouilles doit être considéré comme le principal artisan de la baisse de la piraterie dans la région.

Or l'Etat brille par son absence en Somalie. Même son semblant de façade se fissure depuis les récentes querelles entre le Premier ministre et son Président en réalité dépendant de l'Ethiopie ${ }^{5}$. Dans ces conditions, il parait difficile de lutter efficacement contre la piraterie, une menace maritime qui se combat pourtant à terre. A moyen et long terme, le déploiement des armadas aussi bien occidentales qu'orientales au large de la Somalie ne suffira pas à rassurer la communauté maritime.

\section{Le pirate : une proie patiente, tenace et en voie de reproduction}

Une seconde raison empêche de croire au modèle sud-est asiatique comme exemple parfait à suivre en matière de lutte anti-pirate. Malgré les efforts louables des Etats riverains du détroit de Malacca, rejoints très prochainement par la Thaïlande, les pirates 
rôdent toujours. Le fléau n'est pas encore totalement éradiqué. Le centre d'information ReCAAP (Regional Cooperation Agreement on Combating Piracy and Armed Robbery against Ships in Asia) basé à Singapour mentionne deux zones dangereuses : l'une au large de Belakang Padang, l'autre au large de Mangkai 6 . Il est à noter que ces deux repaires n'ont encore guère profité de l'aide du gouvernement indonésien. Si le premier, au sud de Singapour, est sur le déclin - rapines menées non loin des côtes, par seulement cinq pirates pour chaque attaque - il en va différemment du second, également visité pour des recherches en 2005 et 2006. Situé à la sortie est du détroit, au sud de la mer de Chine méridionale, il est un point de passage obligé, même pour les navires qui contourneraient le détroit de Malacca en empruntant, au sud de Sumatra, celui de la Sonde. Des témoignages ont fait état de pirates thaïlandais mouillant non loin d'une plage déserte, à l'écart du kampung ou petit village - abritant les pêcheurs de l'île. Cet élément était renforcé par la destination des bateaux attaqués, et parfois détournés, vers la Thaïlande. Des recherches plus approfondies devront confirmer - ou non - cette hypothèse.

En conclusion, les patrouilles maritimes, en Insulinde comme vers la Corne de l'Afrique, ne sont pas la panacée mais permettent de parer au plus pressé, voire de créer une psychose chez des pirates peut-être davantage inquiets, méfiants et soucieux. A plus long terme, il devient urgent de trouver une solution plus sociopolitique que strictement militaire. Car la menace gronde et enfle. Le delta du Niger est ainsi frappé par une quarantaine d'attaques par an; ces agressions sont perpétrées par des villageois désoeuvrés contre des bateaux de ravitaillement en route vers les plateformes pétrolières voisines. Un abordage a aussi été recensé au large de la Corse en août dernier. A quand des vols et violences à bord des péniches des quais de Seine?

\section{NOTES}

1. ICC - International Maritime Bureau, January 2008. Piracy and Armed Robbery Against Ships, Annual report, London, 40 p.; ICC - International Maritime Bureau, October 2008. Piracy and Armed Robbery Against Ships, report for the period 1/1-30/9/2008, London, $41 \mathrm{p}$.

2. 17/12/2008. RI rejects U.S. Anti-Piracy Proposal. The Jakarta Post.

3. Energy Information Agency, "World Oil Transit Chokepoints" http://www.eia.doe.gov/cabs/ World_Oil_Transit_Chokepoints/Background.html, January 2008.

4. Fairclough, G, 18/12/2008. Chinese Navy Might Get Role in Piracy Fight. The Wall Street Journal.

5. Gettleman, J, Ibrahim, M, December 16, 2008. Somali president appoints new prime minister. The International Herald Tribune.

6. Information Sharing Centre, November 2008. Report for October 2008, Singapore, ReCAAP, 33 p. 


\section{RÉSUMÉS}

Le second semestre 2008 a été marqué par une explosion des actes de piraterie au large de la Corne de l'Afrique. Les efforts déployés en réaction se sont révélés être si désordonnés qu'ils risquent de ne pas être très efficaces. Plus à l'est, les riverains du détroit de Malacca ont réussi à chasser les pirates. Cependant, ce succès souvent montré en exemple demeure relatif et en aucun cas dû aux seules patrouilles mises en place.

Since last August, there were markedly more attacks registered off the Horn of Africa. The lack of coordination between the numerous initiatives is making surveillance operations inefficient. Further in the east, the littoral States along the Malacca Straits have managed to expel the pirates. Nevertheless, this success - often showed as an example - is not total. Moreover, it is not only due to the sea and air patrols.

\section{INDEX}

Mots-clés : piraterie, marine marchande, Asie du Sud-est, Somalie, Indonésie

Keywords : piracy, shipping, Southeast Asia, Somalia, Indonesia

\section{AUTEUR}

\section{ERIC FRÉCON}

Eric Frécon (isefrecon@ntu.edu.sg) est post-doctorant à la RSIS de Singapour (S. Rajaratnam School of International Studies). Il a notamment publié sur le sujet :

- Frecon, E., à paraître. A la recherche des pirates malais (titre provisoire). Paris, Perrin.

- Frecon, E, 2008. Menace pirate sur les Etats d'Asie du Sud-est, in Huetz de Lemps, Ch, Sevin, O,

L'Asie-Pacifique. Crises et violence. Paris, PUPS, p. 101-123.

- Frecon, E., 2008. Human Security Journal, « Itinéraire de trafiquants : le réseau malais au sud de Singapour », avril 2008. p. 122-129.

- Frecon, E, 2002. Pavillon Noir sur l'Asie du Sud-est : histoire d'une résurgence de la piraterie maritime. Bangkok - Paris, IRASEC - L'Harmattan, 296 p. 